\section{EUS-FNA and biomarkers for the staging of non-small cell lung cancer}

M. Al-Haddad, M. B. Wallace

Division of Gastroenterology and Hepatology, Mayo Clinic College of Medicine, Jacksonville, Florida

\section{Background}

Non-small cell carcinoma of the lung (NSCLC) is the most common cause of cancer death in the United States [1], with more than 160,000 annual deaths [2]. The American Joint Committee on Cancer TNM system is the current staging system for lung cancer, and its goal is to classify patients into groups based on the extent of disease. This system relies heavily on the pathologic evaluation of the primary tumor $(\mathrm{T})$, regional nodes $(\mathrm{N})$ and distant metastases (M). Lymph nodes in the mediastinum are the most common sites of metastases. Recent advances in molecular diagnostics has great potential to increase the accuracy of detection of both primary tumors and metastatic lymph nodes by enabling detection of very small numbers of cancer cells ("micrometastases") using minimally invasive sampling techniques such as image-guided fine needle aspiration (FNA).

Mediastinal lymph nodes evaluation is a critical component of staging NSCLC patients. Lymph nodes larger than $1 \mathrm{~cm}$ in diameter by CT are presumed to contain metastatic disease. However, when compared with surgical pathology, CT misses mediastinal lymph node metastases (false negative) in approximately $13 \%$ of patients and incorrectly suggests it (false positive) in approximately 50\% [3]. Because of this limitation, mediastinoscopy has, for years, been accepted as the "gold standard" for mediastinal staging. Mediastinoscopy requires general anesthesia and specialized expertise to perform safely, and therefore is not universally employed.

Recently, positron emission tomography (PET) has emerged as a non-invasive method for evaluating cancer stage. Recent studies suggest that the sensitivity and specificity of PET may be in excess of $80-90 \%[4,5]$. Because PET relies on the accumulation of a radioactive tracer in proportion to cellular metabolic activity, its ability to detect microscopic tumor deposits and distinguish between inflammation and cancer is limited. Moreover, PET and CT scan cannot make a tissue diagnosis.

The presence or absence of mediastinal lymph node metastases has been classically dependent on histological analysis alone. However, survival statistics indicate clearly that reliance on histology is inadequate. Following presumably curative surgical resection, the five-year survival rate for patients with pathologic stage I disease (no histologic evidence of lymph node metastases) is only $62 \%$. For patients with metastatic disease identified

Correspondence: Michael B. Wallace, M.D. · Associate Professor of Medicine . Division of Gastroenterology and Hepatology · Mayo Clinic Jacksonville · 4500 San Pablo Rd·Jacksonville, FL 32224 ·E-mail: wallace.michael@mayo.edu

Bibliography: Endoscopy 2006; 38 (S1): S114-S117 @ Georg Thieme Verlag KG Stuttgart · New York · ISSN 0013-726X · DOI 10.1055/s-2006-946670 in hilar lymph nodes but not mediastinal lymph nodes (stage II), the five-year survival rate falls to only $42 \%$ [6]. These figures clearly suggest that histological evaluation of mediastinal lymph nodes may miss metastatic disease in a large proportion of patients with NSCLC. Studies have shown that serial sectioning and immunohistochemical staining increase the sensitivity of detection of metastatic disease, and that the presence of metastatic disease detected in this fashion is associated with worse survival [7-9]. Although serial sectioning provides the ability to detect clinically significant metastatic disease, it is extremely time-consuming and expensive and is thus is not practical performed on routine basis.

Standard therapies for patients with NSCLC include surgery, chemotherapy and radiation therapy, depending on the stage of disease. Surgery is considered most appropriate for patients in whom disease is confined to the lung and hilar lymph nodes (stages I and II). For patients with metastatic disease to mediastinal lymph nodes (stage III), the benefit of surgery as primary therapy is questionable and combined chemo-radiotherapy may be most appropriate [10]. Better preoperative staging would reduce the rate of unnecessary surgical exploration and morbidity.

\section{Role of endoscopic ultrasound}

Endoscopic ultrasound (EUS) is a minimally invasive method capable of sampling mediastinal lymph nodes by fine needle aspiration and can be performed under conscious sedation on an outpatient basis. It has been shown that EUS-FNA is a safe and accurate method of staging lymph nodes in the mediastinum of NSCLC patients $[3,11,12]$.

Experience at multiple institutions has shown that EUS-FNA with cytology improves the accuracy of detection of mediastinal metastases compared to CT and PET [3,11,13,15-17]. In addition, EUS-FNA is more cost effective than other surgical methods of lymph node sampling such as mediastinoscopy [14].

Tissue samples obtained by EUS-FNA are typically analyzed by standard cytopathology techniques (thin prep, Papanicolau staining), but can also be analyzed for molecular markers of cancer cells that may not be detectable by cytological methods.

\section{Molecular studies of lymph nodes}

Molecular characterization of lymph node tissue is a rapidly expanding field. Standard cytological or histological methods usually fail to detect early "micrometastases" in lymph nodes. Many of these tumor cells can be detected using molecular analysis for lung epithelial cell specific proteins or mRNA in lymph nodes. Detection of these "micrometastases" using molecular techniques such as immunohistochemistry or reverse transcriptasepolymerase chain reaction ( $\mathrm{rPCR}$ ) has been shown to improve the detection of metastatic epithelial cells in the lymph nodes and bone marrow of patients with lung carcinoma [18-20]. Using this method, several markers have been identified such as telomerase [21] and KS $1 / 4$, which can accurately identify signatures of metastatic disease in cytopathology negative lymph no- 
des [22]. These markers will be discussed in more details in this context. These studies suggest that up to $50 \%$ of patients with histologically normal lymph nodes have micrometastases detected by molecular techniques. The presence of micrometastases has also been shown to have a significant impact on longterm survival even among patients with pathologically "normal" lymph nodes.

\section{Gene marker expression and RT-PCR}

Altered gene expression associated with malignant transformation provides an opportunity to identify the presence of malignant cells by detecting mRNA transcripts that would otherwise not be present in lymph nodes. For example, cytokeratin (CK) genes are normally expressed in epithelial but not lymphoid cells, so the presence of cytokeratin mRNA in a lymph node suggests the presence of metastatic cells of epithelial origin. RT-PCR is highly sensitive technique that allows for detection of rare gene transcripts in tissue samples, and has been reported to be capable of detecting one cancer cell per $10^{6}$ normal cells $[23,24]$. Furthermore, metastatic spread produces ectopic expression of tissue-specific genes.

Clear advantages of RT-PCR include minimal tissue requirements, sensitivity of detection, and potential cost efficiency $[18,25-29]$. Studies with other cancers have shown that RT-PCR is capable of highly sensitive detection of metastases. A good example of this is the application of this technology to improve staging for patients with breast cancer [26]. Similar work with lung cancer has been limited. RT-PCR has been used to detect muc1 transcripts (a mucopolysaccharide gene associated with respiratory epithelium) in histologically negative mediastinal lymph nodes from patients with resected NSCLC [18]. More recently, RT-PCR for p53 and K-ras was shown to improve detection of occult lymph node metastasis compared to immunohistochemistry for cytokeratins [27].

Although yet to be definitely proven for lung cancer, there is considerable evidence to suggest that molecular staging is clinically relevant. An obvious example is hematologic malignancies, where molecular staging has had considerable success. This has been used to monitor residual disease in the peripheral blood and thus guiding the need for further therapy [28-31]. With melanoma, recent studies suggest that RT-PCR is more sensitive than immunohistochemistry for detection of metastatic melanoma in sentinel lymph nodes [32].

Specific genes that are highly expressed in NSCLC have been identified recently. Muc1 was the first marker used for the detection of metastatic NSCLC, but there have been concerns about the specificity of its detection by RT-PCR. With respect to muc1, we found significant overlap in mRNA expression between normal and malignant lymph nodes, suggesting that muc1 lacks specificity in NSCLC. In contrast, the specificity of lunx appeared very high. This result is consistent with Iwao and colleagues [33], who were the first to show that lunx was highly expressed in NSCLC tumors but not in normal lymph nodes. These findings provide evidence that lunx expression might serve as a useful molecular marker for detection of NSCLC lymph node metastases.
Another molecular marker with very high specificity for NSCLC is $K S 1 / 4$, a gene that encodes a glycoprotein expressed on epithelial cells [34]. The protein is recognized by the monoclonal antibody Ber-EP4. Immunohistochemical staining with Ber-EP4 has shown that $K S 1 / 4$ expression is specific for epithelial cells and is present on epithelial cancers (carcinomas) [35]. Interestingly, antibodies against Ber-EP4 have shown promise in clinical trials for colorectal cancer. In a study of 189 patients with resected stage III colorectal cancer, treatment with edrecolomab (an antibody to BerEP4) resulted in a $32 \%$ increase in overall survival compared with no treatment $(\mathrm{P}<0.01)$, and decreased the tumor recurrence rate by $23 \%(\mathrm{P}<0.04)[36]$. These results raise the possibility that edrecolomab therapy may have activity in NSCLC patients.

\section{Telomerase expression}

Telomerase is an enzyme expressed in the rapidly dividing cancerous cells of the lung. Activation of the telomerase gene has been analyzed using a variety of techniques. Most commonly, the activity of the telomerase is measured by using telomerase harvested from the cell extract to add telomeric repeats to the end of a primer followed by amplification of the product using the polymerase chain reaction (TRAP). However, this technique requires approximately $10^{3}$ cells [37]. Because of the small size of the samples, and potentially the few malignant cells in samples obtained by EUS, human telomerase catalytic subunit gene (hTERT) and RT-PCR have been used to detect molecular evidence of malignant lymph nodes in lung cancer [21]. hTERT encodes a protein that is the rate-limiting determinant of the enzymatic activity of human telomerase and expression appears to develop early in the course of tumorigenesis [38]. In addition, expression of this gene has been shown to correlate with telomerase activity $[39,40]$, metastatic disease [41], and shorter survival in non-small cell lung cancer $[40,42,43]$.

Evidence of telomerase over-expression was noted in both pathologically positive and negative mediastinal lymph nodes from patients with NSCLC after EUS-FNA, and the absence of telomerase expression in all normal control lymph nodes. Almost one third of pathologically negative lymph nodes over-express telomerase. The clinical relevance of these findings remains to be determined by survival studies, but the pattern of expression suggests that analysis of hTERT expression may prove useful in detecting micrometastatic disease. It does not appear that rtPCR "positivity" in some histologically negative lymph nodes is predictive of other histologically positive lymph nodes at the time of complete surgical lymphadenectomy.

Telomerase represents a unique target for molecular staging in lung cancer. Not only is telomerase expressed in the majority of lung cancers, but also by all histologic types of lung cancer, and expression in non-small cell lung cancer has been shown to be independently predictive of a worse prognosis [44]. However, recent studies in animal models of lung inflammation have put into question the specificity of telomerase as marker of malignancy. Injury to rat lungs caused by both silica and bleomycin have been shown to be associated with the development of increased telomerase activity in lung fibroblasts $[45,46]$, raising 
the question as to whether telomerase might be increased in lungs inflamed by other agents such as cigarette smoke. Yet in two studies of patients with known non-small cell lung cancer analysis of tumor normal pairs showed no evidence of telomerase gene expression in any of the paired normal specimens (total of 218 samples for both studies) [44,47]. Evaluation of lymph nodes for the presence of metastatic disease using telomerase remains controversial. While high levels of telomerase activity in hyperplastic lymph nodes and tonsils have been reported [48], others have found a significant difference in telomerase activity between benign and malignant lymph nodes with high levels of activity in malignant and only low levels of activity in benign nodes [49].

These data suggest that detection of hTERT gene expression in lymph nodes may identify micrometastatic disease when normal cytologic and histologic techniques fail. Further studies are needed to determine the clinical significance of expression of hTERT in pathologically negative lymph nodes on survival, and whether chemo-radiotherapy is beneficial to patients with rtPCR evidence but not histological evidence of mediastinal lymph node metastases (rtPCR stage III).

\section{EGFR gene: A new target}

The epidermal growth factor receptor family of genes (EGFR) encodes widely expressed transmembrane molecules that have been implicated in the development and progression of cancer. The EGFR gene is frequently expressed in solid tumors, and this may correlate with a poor clinical outcome in certain cancers [50].

Non-small-cell lung cancer frequently expresses EGFR [51 - 53], and for this reason, it is of considerable interest for clinical trials of inhibitors of the tyrosine kinase of EGFR [54]. The kinase inhibitors erlotinib (Tarceva, OSI Pharmaceuticals, Melville, NY, USA) and gefitinib (Iressa, AstraZeneca, Wilmington, DE, USA) have been studied most extensively in clinical trials [54-56]. Somatic mutations in the region of EGFR that encodes the tyrosine kinase domain of the receptor (exons 18 through 21) have been identified in lung cancer [57,58], and many studies suggest that they can be used to predict responsiveness to gefitinib and erlotinib [57-63]. Risk factors for such mutations include female gender, Asians, and patients who have never smoked.

A recent study evaluated the effect of EGFR expression in NSCLC on responsiveness to erlotinib and survival [64]. Patients with EGFR mutation were found to be more responsive to this agent, though it did not demonstrate a significant survival benefit compared to the placebo group. Further studies are underway to confirm this effect on survival. It remains to be determined if mutational analysis is necessary to identify patients in whom treatment with EGFR inhibitors is appropriate.

\section{Conclusion}

There in increasing evidence that molecular diagnostic methods allow detection of tumor specific genes in lung cancer speci- mens. The most promising methods include real time reverse transcriptase PCR in conjunction with minimally invasive fine needle aspiration samples of normal lymph nodes in lung cancer, patients. Promising markers for detection of micrometastases to lymph nodes include $\mathrm{KS}^{1 / 4}$ and telomerase. Current research is evaluating the effect of EGFR mutations on response to treatment and survival.

Several important issues remain unanswered though. Since overexpression alone is not a definitive evidence of micrometastatic disease, the presence of these markers needs to be correlated directly with clinical outcome such as disease recurrence and survival. However, the most promising aspect of this technology is the broad applicability to other tumors. Combining the ability to easily access tissue with EUS-FNA and modern genomic and proteomic methods offers a rich area for further research and clinical applications.

\section{References}

${ }^{1}$ Ries LAG, E M, Kosary CL, Hankey BF, Miller BA, Clegg L, Edwards BK. SEER Cancer Statistics Review. 2002

2 Landis SH et al. Cancer statistics, 1999. CA Cancer J Clin 1999; 49 (1): $8-31$

${ }^{3}$ Wallace MB et al. Endoscopic ultrasound-guided fine needle aspiration for staging patients with carcinoma of the lung. Ann Thorac Surg 2001; 72 (6): $1861-1867$

${ }^{4}$ Gupta NC et al. Mediastinal lymph node sampling following positron emission tomography with fluorodeoxyglucose imaging in lung cancer staging. Chest 2001; 120 (2): $521-527$

${ }^{5}$ Dwamena BA et al. Metastases from non-small cell lung cancer: mediastinal staging in the 1990s-meta-analytic comparison of PET and CT. Radiology 1999; 213 (2): 530-536

${ }^{6}$ Mountain CF, Dresler CM. Regional lymph node classification for lung cancer staging. Chest 1997; 111 (6): $1718-1723$

${ }^{7}$ Dobashi K et al. Micrometastatic P53-positive cells in the lymph nodes of non-small-cell lung cancer: prognostic significance. J Thorac Cardiovasc Surg 1997; 114 (3): 339-346

${ }^{8}$ Dowlatshahi K et al. Lymph node micrometastases from breast carcinoma: reviewing the dilemma. Cancer 1997; 80 (7): 1188-1197

${ }^{9}$ Izbicki JR et al. Prognostic value of immunohistochemically identifiable tumor cells in lymph nodes of patients with completely resected esophageal cancer. N Engl J Med 1997; 337 (17): 1188-1194

${ }^{10}$ Miller JD, Gorenstein LA, Patterson GA. Staging: the key to rational management of lung cancer. Ann Thorac Surg 1992; 53 (1): 170-178

${ }^{11} \mathrm{Gress}$ FG et al. Endoscopic ultrasonography, fine-needle aspiration biopsy guided by endoscopic ultrasonography, and computed tomography in the preoperative staging of non-small-cell lung cancer: a comparison study. Ann Intern Med 1997; 127 (8 Pt 1): 604-612

12 Wiersema MJ et al. Preoperative staging of non-small cell lung cancer: transesophageal US-guided fine-needle aspiration biopsy of mediastinal lymph nodes. Radiology 1994; 190 (1): 239-342

${ }^{13}$ Fritscher-Ravens A et al. Mediastinal lymph node involvement in potentially resectable lung cancer: comparison of $\mathrm{CT}$, positron emission tomography, and endoscopic ultrasonography with and without fineneedle aspiration. Chest 2003; 123 (2): 442 - 451

${ }^{14}$ Harewood GC et al. Cost-minimization analysis of alternative diagnostic approaches in a modeled patient with non-small cell lung cancer and subcarinal lymphadenopathy. Mayo Clin Proc 2002; 77 (2): 155 164

${ }^{15}$ Gress FG et al. Endoscopic ultrasonography, fine-needle aspiration biopsy guided by endoscopic ultrasonography, and computed tomography in the preoperative staging of non-small-cell lung cancer: a comparison study [see comments]. Ann Intern Med 1997; 127 (8 Pt 2): $604-612$

${ }^{16}$ Silvestri GA et al. Endoscopic ultrasound with fine-needle aspiration in the diagnosis and staging of lung cancer. Ann Thorac Surg 1996; 61 (5): 1441 -5; discussion 1445-1446 
${ }^{17}$ Wiersema MJ et al. Preoperative staging of non-small cell lung cancer: transesophageal US-guided fine-needle aspiration biopsy of mediastinal lymph nodes. Radiology 1994; 190 (1): 239-242

${ }^{18}$ Salerno CT et al. Detection of occult micrometastases in non-small cell lung carcinoma by reverse transcriptase-polymerase chain reaction. Chest 1998; 113 (6): 1526-1532

${ }^{19}$ Maruyama R et al. Relationship between early recurrence and micrometastases in the lymph nodes of patients with stage I non-small-cell lung cancer. J Thorac Cardiovasc Surg 1997; 114 (4): 535-543

${ }^{20}$ Chen ZL et al. Frequency and distribution of occult micrometastases in lymph nodes of patients with non-small-cell lung carcinoma. J Natl Cancer Inst 1993; 85 (6): $493-498$

${ }^{21}$ Wallace MB et al. Detection of telomerase expression in mediastinal lymph nodes of patients with lung cancer. Am J Respir Crit Care Med 2003; 167 (12): $1670-1675$

${ }^{22}$ Wallace MB et al. Accurate molecular detection of non-small cell lung cancer metastases in mediastinal lymph nodes sampled by endoscopic ultrasound-guided needle aspiration. Chest 2005; 127 (2): 430 - 437

${ }^{23}$ Mori $\mathrm{M}$ et al. Detection of cancer micrometastases in lymph nodes by reverse transcriptase-polymerase chain reaction. Cancer Res 1995; 55 (15): $3417-3420$

${ }^{24}$ Noguchi $S$ et al. Detection of breast cancer micrometastases in axillary lymph nodes by means of reverse transcriptase-polymerase chain reaction. Comparison between MUC1 mRNA and keratin 19 mRNA amplification. Am J Pathol 1996; 148 (2): 649-656

${ }^{25}$ Lockett MA et al. Efficacy of reverse transcriptase-polymerase chain reaction screening for micrometastic disease in axillary lymph nodes of breast cancer patients. Am Surg 1998; 64 (6): 539-43; discussion $543-454$

${ }^{26}$ Mitas M et al. Quantitative real-time RT-PCR detection of breast cancer micrometastasis using a multigene marker panel. Int J Cancer 2001; 93 (2): $162-171$

${ }^{27}$ Hashimoto T et al. Prognostic value of genetically diagnosed lymph node micrometastasis in non-small cell lung carcinoma cases. Cancer Res 2000; 60 (22): 6472-6478

${ }^{28}$ Pallisgaard $\mathrm{N}$ et al. Rapid and sensitive minimal residual disease detection in acute leukemia by quantitative real-time RT-PCR exemplified by $\mathrm{t}(12 ; 21)$ TEL-AML1 fusion transcript. Genes Chromosomes Cancer 1999; 26 (4): 355-365

${ }^{29}$ Mensink E et al. Quantitation of minimal residual disease in Philadelphia chromosome positive chronic myeloid leukaemia patients using real-time quantitative RT-PCR. Br J Haematol 1998; 102 (3): $768-774$

${ }^{30}$ Emig $\mathrm{M}$ et al. Accurate and rapid analysis of residual disease in patients with CML using specific fluorescent hybridization probes for real time quantitative RT-PCR. Leukemia 1999; 13 (11): 1825-1832

${ }^{31}$ Cassinat B et al. Quantitation of minimal residual disease in acute promyelocytic leukemia patients with $\mathrm{t}(15 ; 17)$ translocation using realtime RT-PCR. Leukemia 2000; 14 (2): 324- 328

32 Bostick PJ et al. Limitations of specific reverse-transcriptase polymerase chain reaction markers in the detection of metastases in the lymph nodes and blood of breast cancer patients. J Clin Oncol 1998; 16 (8): $2632-2640$

${ }^{33}$ Iwao $\mathrm{K}$ et al. Isolation of a novel human lung-specific gene, LUNX, a potential molecular marker for detection of micrometastasis in nonsmall-cell lung cancer. Int J Cancer 2001; 91 (4): 433-437

34 Perez MS, Walker LE. Isolation and characterization of a cDNA encoding the KS1/4 epithelial carcinoma marker. J Immunol 1989; 142 (10): $3662-3667$

${ }^{35}$ Latza U et al. Ber-EP4: new monoclonal antibody which distinguishes epithelia from mesothelial. J Clin Pathol 1990; 43 (3): 213 - 219

${ }^{36}$ Schwartzberg LS. Clinical experience with edrecolomab: a monoclonal antibody therapy for colorectal carcinoma. Crit Rev Oncol Hematol 2001; 40 (1): $17-24$

${ }^{37} \mathrm{Kim}$ NW et al. Specific association of human telomerase activity with immortal cells and cancer. Science 1994; 266 (5193): 2011 - 2015

38 Kolquist KA et al. Expression of TERT in early premalignant lesions and a subset of cells in normal tissues. Nat Genet 1998; 19 (2): 182-186

${ }^{39}$ Saretzki G et al. hTERT gene dosage correlates with telomerase activity in human lung cancer cell lines. Cancer Lett 2002; 176 (1): 81 -91

${ }^{40}$ Marchetti A et al. Prediction of survival in stage I lung carcinoma patients by telomerase function evaluation. Lab Invest 2002; 82 (6): $729-736$
${ }^{41}$ Toomey D et al. Immune function, telomerase, and angiogenesis in patients with primary, operable nonsmall cell lung carcinoma: tumor size and lymph node status remain the most important prognostic features. Cancer 2001; 92 (10): $2648-2657$

${ }^{42} \mathrm{Hara} \mathrm{H}$ et al. Clinicopathologic significance of telomerase activity and hTERT mRNA expression in non-small cell lung cancer. Lung Cancer 2001; 34 (2): 219-226

${ }^{43}$ Komiya T et al. Prognostic significance of hTERT expression in nonsmall cell lung cancer. Int J Oncol 2000; 16 (6): 1173-1177

${ }^{44}$ Taga $\mathrm{S}$ et al. Prognostic impact of telomerase activity in non-small cell lung cancers. Ann Surg 1999; 230 (5): $715-720$

${ }^{45} \mathrm{Kim}$ JK et al. Activation of telomerase by silica in rat lung. Toxicol Lett 2000; 111 (3): $263-270$

${ }^{46}$ Nozaki $Y$ et al. Induction of telomerase activity in fibroblasts from bleomycin-injured lungs. Am J Respir Cell Mol Biol 2000; 23 (4): $460-465$

${ }^{47}$ Kumaki F et al. Telomerase activity and expression of human telomerase RNA component and human telomerase reverse transcriptase in lung carcinomas. Hum Pathol 2001; 32 (2): 188-195

${ }^{48}$ Xinarianos $\mathrm{G}$ et al. Evaluation of telomerase activity in bronchial lavage as a potential diagnostic marker for malignant lung disease. Lung Cancer 2000; 28 (1): 37-42

${ }^{49}$ Sumida T et al. Clinical usefulness of telomerase assay for the detection of lymph node metastasis in patients with oral malignancy. Arch Pathol Lab Med 2000; 124 (3): $398-400$

${ }^{50}$ Nicholson RI, Gee JM, Harper ME. EGFR and cancer prognosis. Eur J Cancer 2001; 37 Suppl 4: S9-15

${ }^{51}$ Brabender J et al. Epidermal growth factor receptor and HER2-neu mRNA expression in non-small cell lung cancer Is correlated with survival. Clin Cancer Res 2001; 7 (7): 1850-1855

52 Selvaggi $G$ et al. Epidermal growth factor receptor overexpression correlates with a poor prognosis in completely resected non-small-cell lung cancer. Ann Oncol 2004; 15 (1): 28 - 32

53 Swinson DE, Cox G, O'Byrne KJ. Coexpression of epidermal growth factor receptor with related factors is associated with a poor prognosis in non-small-cell lung cancer. Br J Cancer 2004; 91 (7): 1301 - 1307

${ }^{54}$ Sridhar SS, Seymour L, Shepherd FA. Inhibitors of epidermal-growthfactor receptors: a review of clinical research with a focus on nonsmall-cell lung cancer. Lancet Oncol 2003; 4 (7): 397-406

${ }^{55}$ Kris MG et al. Efficacy of gefitinib, an inhibitor of the epidermal growth factor receptor tyrosine kinase, in symptomatic patients with non-small cell lung cancer: a randomized trial. Jama 2003; 290 (16): $2149-2158$

${ }^{56}$ Perez-Soler R et al. Determinants of tumor response and survival with erlotinib in patients with non-small-cell lung cancer. J Clin Oncol 2004; 22 (16): $3238-3247$

${ }^{57}$ Lynch TJ et al. Activating mutations in the epidermal growth factor receptor underlying responsiveness of non-small-cell lung cancer to gefitinib. N Engl J Med 2004; 350 (21): 2129-2139

58 Paez JG et al. EGFR mutations in lung cancer: correlation with clinical response to gefitinib therapy. Science 2004; 304 (5676): 1497-1500

${ }^{59}$ Cappuzzo $\mathrm{F}$ et al. Epidermal growth factor receptor gene and protein and gefitinib sensitivity in non-small-cell lung cancer. J Natl Cancer Inst 2005; 97 (9): $643-655$

${ }^{60}$ Marchetti A et al. EGFR mutations in non-small-cell lung cancer: analysis of a large series of cases and development of a rapid and sensitive method for diagnostic screening with potential implications on pharmacologic treatment. J Clin Oncol 2005; 23 (4): 857-865

61 Shigematsu $\mathrm{H}$ et al. Clinical and biological features associated with epidermal growth factor receptor gene mutations in lung cancers. J Natl Cancer Inst 2005; 97 (5): 339-346

${ }^{62}$ Yang SH et al. Mutations in the tyrosine kinase domain of the epidermal growth factor receptor in non-small cell lung cancer. Clin Cancer Res 2005; 11 (6): 2106-2110

${ }^{63} \mathrm{Kim} \mathrm{KS}$ et al. Predictors of the response to gefitinib in refractory nonsmall cell lung cancer. Clin Cancer Res 2005; 11 (6): 2244-2251

${ }^{64}$ Tsao MS et al. Erlotinib in lung cancer - molecular and clinical predictors of outcome. N Engl J Med 2005; 353 (2): 133-144 